\section{The discovery of the brain lymphatic system}

\section{Paolo Zamboni \\ Translational Surgery and Vascular Diseases Center, University of Ferrara, Italy}

In the latest issue of Nature Antoine Louveau and a team of collaborators of the University of Virginia, published a breakthrough article for who is interested in lymphatics, with a clear and elegant description of the lymphatic system of the brain. ${ }^{1}$

Surprisingly, few days later a group of Helsinki confirmed with other experiments the existence of a cerebral lymphatics network connected with cervical nodes. ${ }^{2}$

Despite the rapid popularity of this article it does not represent a revolutionary discovery, but rather an updated systematic description of connections and function of the lymphatic system in this particular anatomical district. It should be, in fact, recognized that a forgotten literature on Durand Fardel's periarterial and perivenous Virchow-Robin's spaces, recently turned into glymphatic system, ${ }^{3}$ exists from more than a century. However, this article permits to definitely overcome the neurocentric concept that lymphatic drainage of the central nervous system is confined to the meningeal

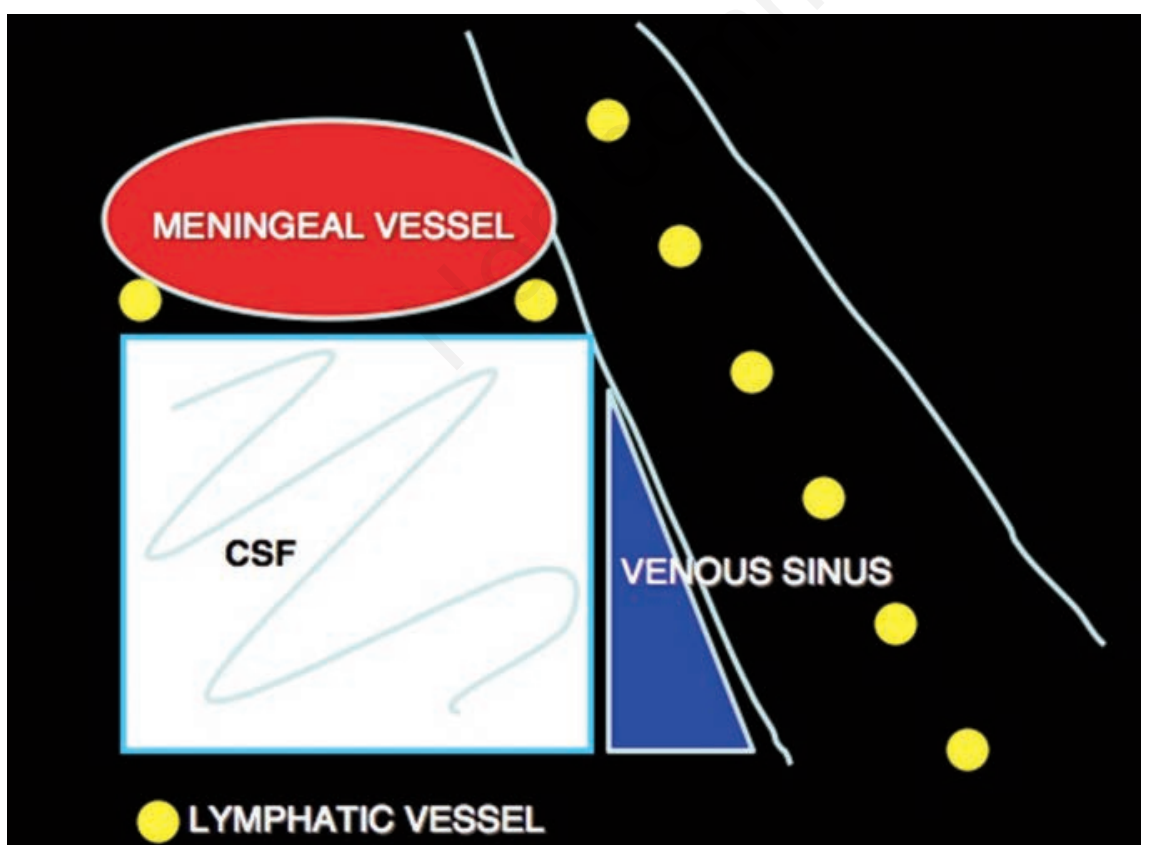

Figure 1. Schematic representation of the functional histology specimen showed in the Nature article. ${ }^{1}$ The lymphatic system lines the venous dural sinuses and is closely interconnected with cerebrospinal fluid (CSF) space, as well as with meningeal vessels. Out of the skull the interconnection was found by the researchers of the Virginia University both with the internal jugular vein and with the deep cervical lymph-nodes. compartment and there is a lack of lymphatic drainage in the brain parenchyma. The Authors also better clarify the structure and the function of the so called glymphatic system, till now more supposed than proved. ${ }^{3}$

The group of the University of Virginia clearly found the lymphatic system practically in the wall of the dural veins, and this testifies the functional and anatomical cross talk between the two systems, something which gives the title to our Journal. Moreover, they demonstrated with elegant images that interconnection of the lymphatic collectors are not just veins, but also the cerebrospinal fluid (CSF) (Figure 1), and the deep cervical lymphnodes and with the internal jugular veins, as well. What are the potential implications of this discovery? The main consequence is that the dogma of the immune privilege of the brain, as admitted by the Authors themselves, dramatically falls down. We all studied that the brain is segregated from the immunitary point of view. ${ }^{4}$ The physical reason was just the lack of lymphatics, which means no T-cell gataway into and out of the brain. The demonstration of the central nervous system lymphatics warrants an urgent reassessment of the basic assumption in neuroimmunology.

In fact, the immune privilege of the brain means that T-cell and antibodies found in the brain and/or in the CSF are of brain autochthonous production. Consequently, the presence of T-cells was considered of autoimmune origin. of the brain lymphatics with the deep cervical lymph-nodes, a draining route toward the thoracic duct, be improved? Both articles really open new and stimulating questions, and this happens only when a discovery is a real scientific advancement.

\section{References}

1. Louveau A, Smirnov I, Keyes TJ, et al. Structural and functional features of central nervous system lymphatic vessels. Nature 2015 [Epub ahead of print].

2. Aspelund A, Antila S, Proulx ST, et al. A dural lymphatic vascular system that drains brain interstitial fluid and macromolecules. J Exp Med 2015 [Epub ahead of print]

3. Yang L, Kress BT, Weber HJ, et al. Evaluating glymphatic pathway function utilizing clinically relevant intrathecal infusion of CSF tracer. J Transl Med 2013;11:107.

4. Antel J, Birnbaum G, Hartung H-P, eds. Clinical neuroimmunology. Boston: Blackwell Science; 1998. 